\title{
Patients with chronic gastrointestinal ischemia have a higher cardiovascular disease risk and mortality
}

\author{
Aria Sana ${ }^{a, *}$, Désirée van Noord ${ }^{a, 1}$, Peter B.F. Mensink ${ }^{a, 1}$, Stephanie Kooij ${ }^{b, 2}$, Kim van Dijk ${ }^{b, 2}$, \\ Bert Bravenboer $^{c, 3}$, Aloysius G. Lieverse ${ }^{\mathrm{d}, 4}$, Eric J.G. Sijbrands ${ }^{\mathrm{b}, 2}$, Janneke G. Langendonk ${ }^{\mathrm{b}, 2}$, \\ Ernst J. Kuipers a,b,1 \\ ${ }^{a}$ Departments of Gastroenterology and Hepatology, Erasmus Medical Center, Rotterdam, The Netherlands \\ ${ }^{\mathrm{b}}$ Department of Internal Medicine, Erasmus Medical Center, Rotterdam, The Netherlands \\ ${ }^{\mathrm{c}}$ Department of Internal Medicine, Catharina Ziekenhuis, Eindhoven, The Netherlands \\ ${ }^{\mathrm{d}}$ Department of Internal Medicine, Maxima Medisch Centrum, Eindhoven, The Netherlands
}

\section{A R T I C L E I N F O}

\section{Article history:}

Received 23 February 2012

Received in revised form

28 June 2012

Accepted 6 July 2012

Available online 15 July 2012

Keywords:

Atherosclerosis

Chronic gastrointestinal ischemia

Cardiovascular disease

Mortality

\begin{abstract}
A B S T R A C T
Objectives: We determined the prevalence of classical risk factors for atherosclerosis and mortality risk in patients with CGI.

Methods: A case-control study was conducted. Patients referred with suspected CGI underwent a standard work-up including risk factors for atherosclerosis, radiological imaging of abdominal vessels and tonometry. Cases were patients with confirmed atherosclerotic CGI. Controls were healthy subjects previously not known with CGI. The mortality risk was calculated as standardized mortality ratio derived from observed mortality, and was estimated with ten-year risk of death using SCORE and PREDICT. Results: Between 2006 and 2009, 195 patients were evaluated for suspected CGI. After a median followup of 19 months, atherosclerotic CGI was diagnosed in 68 patients. Controls consisted of 132 subjects. Female gender, diabetes, hypercholesterolemia, a personal and family history of cardiovascular disease (CVD), and current smoking are highly associated with CGI. After adjustment, female gender (OR 2.14 95\% CI 1.05-4.36), diabetes (OR 5.59, 95\% CI 1.95-16.01), current smoking (OR 5.78, 95\% CI 2.27-14.72), and history of CVD (OR 21.61, 95\% CI 8.40-55.55) remained significant. CGI patients $>55$ years had a higher median ten-year risk of death ( $15 \%$ vs. $5 \%, P=0.001)$ compared to controls. During follow-up of 116 person-years, standardized mortality rate was higher in CGI patients (3.55; 95\% CI 1.70-6.52).

Conclusions: Patients with atherosclerotic CGI have an increased estimated CVD risk, and severe excess mortality. Secondary cardiovascular prevention therapy should be advocated in patients with CGI.
\end{abstract}

(C) 2012 Elsevier Ireland Ltd. All rights reserved.
Abbreviations: BMI, body mass index; CACS, celiac artery compression syndrome; CGI, chronic gastrointestinal ischemia; CTA, computed tomography angiography; IQR, interquartile range; MRA, magnetic resonance angiography; NOMI, non-occlusive mesenteric ischemia; SMR, standardized mortality ratio.

* Corresponding author. Erasmus MC, University Medical Center, Department of Gastroenterology and Hepatology, Room L-227, 's-Gravendijkwal 230, 3015 CE Rotterdam, The Netherlands. Tel.: +31 1070 30212; fax: +31 107032793.

E-mail addresses: a.sana@erasmusmc.nl (A. Sana), d.vannoord@erasmusmc.nl (D. van Noord), p.mensink@erasmusmc.nl (P.B.F. Mensink), stephaniekooij@ tiscali.nl (S. Kooij), kimvandijk1@upcmail.nl (K. van Dijk), bert.bravenboer@ catharina-ziekenhuis.nl (B. Bravenboer), L.Lieverse@mmc.nl (A.G. Lieverse), e.sijbrands@erasmusmc.nl (E.J.G. Sijbrands), j.langendonk@erasmusmc.nl (J.G. Langendonk), e.j.kuipers@erasmusmc.nl (E.J. Kuipers).

1 Room Ba-393, 's-Gravendijkwal 230, 3015 CE Rotterdam, The Netherlands.

2 Room D-431, 's-Gravendijkwal 230, 3015 CE Rotterdam, The Netherlands.

3 Catharina Ziekenhuis, Postbus 1350, 5602 ZA Eindhoven, The Netherlands.

4 Maxima Medische Centrum, Postbus 90052, 5600 PD Eindhoven, The Netherlands.

\section{Introduction}

Chronic gastrointestinal ischemia (CGI) is in the majority of cases caused by narrowing of the gastrointestinal arteries, with atherosclerosis being the most common underlying cause [1,2]. Subsequent cardiovascular disease (CVD) preventive therapy is not considered standard care.

The conventional risk factors for atherosclerosis are: male gender, age, diabetes mellitus, smoking, hypertension, hypercholesterolemia, medical history of previous CVD, positive family history of (premature) CVD, obesity, and physical inactivity $[3,4]$. Any ischemic event is a strong independent risk factor for a new cardiovascular event [5-11].

Although CGI is considered a manifestation of atherosclerosis, studies focused on a cardiovascular work-up of classical risk factors for atherosclerosis in patients with CGI are missing. Previous case 
series have reported inconsistent findings. A wide range of distribution of classical risk factors for atherosclerosis has been reported in the baseline characteristics of case series: a 10-93\% prevalence of hypercholesterolemia, $64-92 \%$ of hypertension, and $36-72 \%$ of previous coronary artery disease $[1,12-18]$. The primary objective of this case-control study was to determine the contribution of classical atherosclerotic risk factors to atherosclerotic CGI, and the secondary objective was to assess the mortality risk in treated patients.

\section{Methods}

A case-control study was conducted. The Institutional Review Boards of the Erasmus Medical Center and the Eindhoven hospitals approved the study. Only patients with written informed consent entered the present study. Control subjects from the DiaGene Study population served as controls [19]. DiaGene is a case-control study, including patients with diabetes (cases) and subjects without a previous history of diabetes (controls). The DiaGene is conducted in three general hospitals in Eindhoven, The Netherlands, Controls were recruited via an advertisement in different local news papers. Subjects could participate in the study if they were not known with diabetes. Upon registration and after giving their written informed consent, they received an invite to one of the participating medical centers. They completed a questionnaire concerning cardiovascular risk assessment, medical and family history for CVD, medication use, and smoking. Fasting blood samples were drawn and body mass index and blood pressure were determined. After obtaining all the information concerning cardiovascular risk assessment, there was no further follow-up of the controls. In the current study, we included the first 132 consecutive subjects who responded for the DiaGene study as controls. None of these controls were diagnosed with CGI.

\subsection{Case selection}

Consecutive patients with suspected CGI referred to our tertiary center for evaluation were considered for inclusion. Patients with intravascular atherosclerotic CGI were included as cases. The full description of the selection of the cases is described below.

\subsection{Diagnostic work-up including cardiovascular diagnostic work-up}

All patients referred with suspected CGI underwent a thorough medical and physical examination, including ECG and with extensive questioning using a structured questionnaire concerning cardiovascular risk assessment, clinical complaints, weight before onset of complaints, medical and family history for CVD. During physical examination, height and weight were measured. From all patients referred with suspected CGI, venous blood samples were obtained after 9-h fasting for measurement of serum cholesterol, triglycerides, LDL-cholesterol, HDL-cholesterol, and blood glucose. Documented coronary artery disease included at least one of the following criteria: history of cardiac angina, myocardial infarction (MI), percutaneous coronary intervention $(\mathrm{PCI})$, or coronary artery bypass graft surgery (CABG). Documented cerebrovascular accident included a diagnosis of transient ischemic attack (TIA) or ischemic stroke. Documented peripheral arterial disease consisted of intermittent claudication previous treatment such as angioplasty, endovascular stent placement, peripheral arterial bypass graft or atherectomy. Hypercholesterolemia was considered present when subjects reported hypercholesterolemia, and/or used a statin in the absence of CVD, and/or when LDL-cholesterol or total cholesterol was above the 95th percentile for age and gender. Hypertension was defined as known with hypertension or using anti- hypertensive medication or a systolic blood pressure $>140 \mathrm{mmHg}$ or a diastolic blood pressure $>90 \mathrm{mmHg}$. Diabetes mellitus was defined according to WHO criteria or use of blood glucose lowering agents.

Next to thorough medical and physical examination, all patients suspected for CGI underwent radiological evaluation by means of abdominal CT- or MR angiography, and/or conventional angiography to visualize the gastrointestinal arteries (celiac artery, superior mesenteric artery and inferior mesenteric artery) in combination with functional testing for mucosal perfusion by means of a 24-h gastric and jejunal tonometry as described before $[2,20,21]$.

\subsection{Consensus diagnosis and definite diagnosis of CGI}

Medical history, complaints, and the results of all diagnostic procedures were discussed in a dedicated multidisciplinary team consisting of a vascular surgeon, intervention radiologist and gastroenterologist, all specialized in CGI [21]. The discussion resulted in a final expert-based consensus diagnosis of CGI or nonCGI. The consensus diagnosis of CGI was made in presence of 1) a clinical suspicion of CGI, 2 ) a significant stenosis (>70\%) in at least one of the gastrointestinal arteries, and 3) detection of mucosal ischemia by means of 24-h gastric and jejunal tonometry. Patients with a consensus diagnosis of CGI were classified as having either occlusive or non-occlusive CGI. Non-occlusive CGI, also called nonocclusive mesenteric ischemia (NOMI), was defined as gastrointestinal ischemia in the absence of significant vascular stenosis. Patients with occlusive disease were classified as either having an intravascular stenosis, or extravascular compression due to the celiac artery compression syndrome (CACS) (see Fig. 1). Patients diagnosed with NOMI, vascular arteritis and CACS were excluded from the present analyses. All remaining patients with CGI were diagnosed with intravascular stenotic or atherosclerotic CGI. These patients were considered eligible for endovascular or surgical revascularization. All treated patients were prospectively followed at the out-patient clinic with regular visits [21]. The definite diagnosis of atherosclerotic CGI was made after persistent relief of symptoms during long-term follow-up of at least 12 months after initiation of therapy [21]. Patients with definite atherosclerotic CGI were included as cases in the current study.

\subsection{Estimation of ten years risk of death due to cardiovascular disease}

The ten-year mortality risk was estimated in patients without a history of cardiovascular event, with the SCORE risk system, and for patients with previous MI and CABG the PREDICT risk score was used. The PREDICT risk score has been developed for estimating the 6-year mortality risk after hospitalization for acute MI or unstable angina. It is based on factors such as age, ECG at the time of hospitalization, comorbidities and renal function. There is no risk score for estimating the ten-year risk of death due to cardiovascular event for patients with peripheral artery disease or with a cerebrovascular event in the past. We estimated the ten-year mortality in patients with a history of stroke or TIA by using the risk of death at ten years for these patients as described in the DUTCH transient ischemic Trial [22].

\subsection{Baseline measurements}

Venous blood was sampled after 9-h fasting for measurement of serum cholesterol, triglycerides, LDL-cholesterol, HDL-cholesterol, and blood glucose. Kidney function was categorized according to 


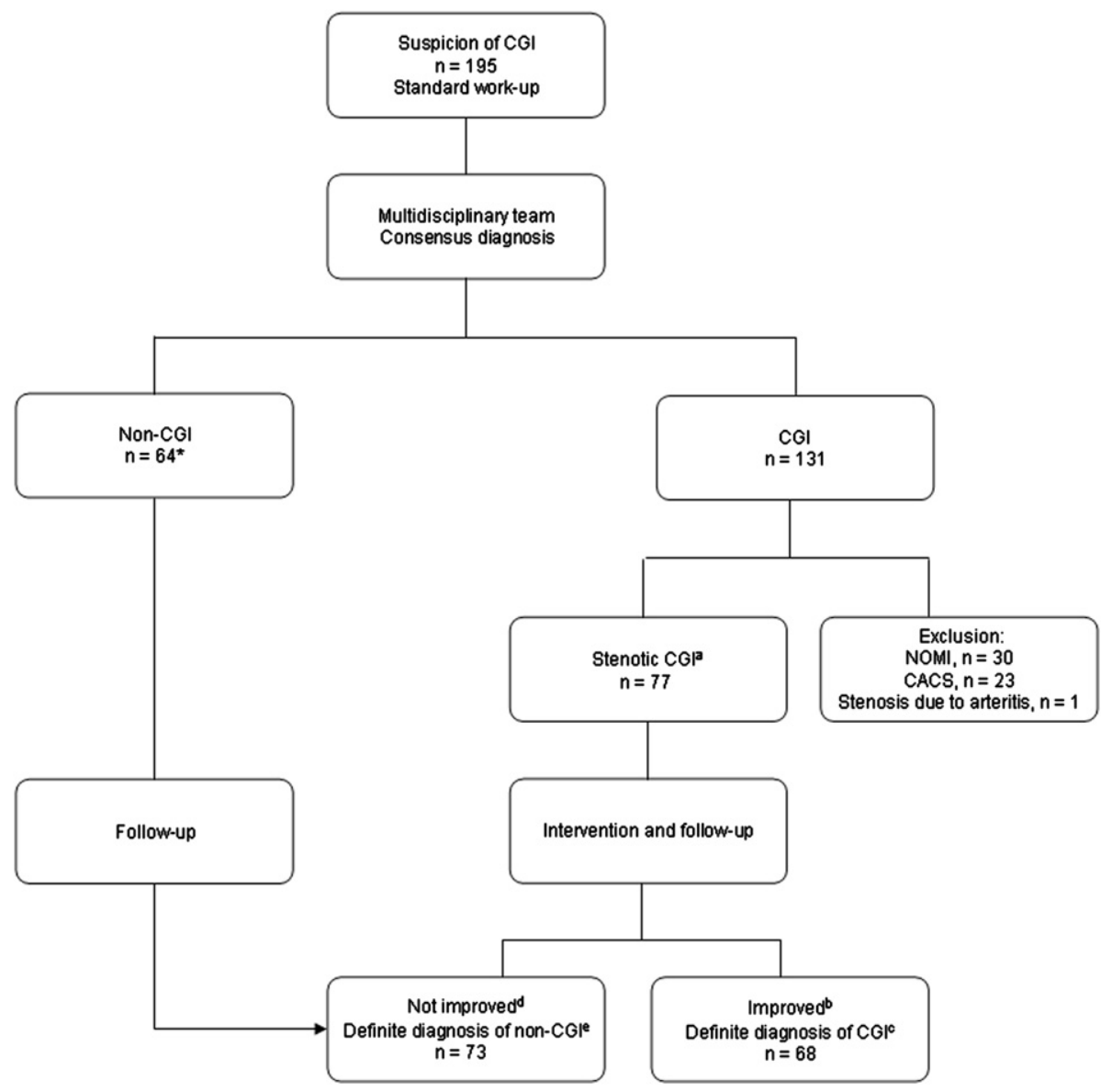

Fig. 1. Flow chart of the study

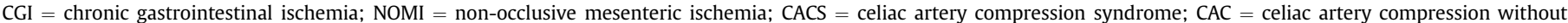
ischemia.

${ }^{\mathrm{a}}$ stenotic CGI = CGI in presence of intravascular atherosclerotic stenosis; treated with endovascular stent placement or surgery.

bymptoms improvement.

${ }^{c}$ A definite diagnosis of CGI was made if a patient was free of symptoms after adequate therapy for at least 12 months of follow-up;

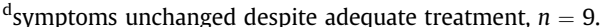

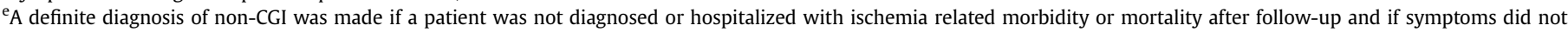
improve despite adequate treatment.

${ }^{*}$ CAC, $n=7$.

classification established by the National Foundation Kidney Disease Outcomes Quality Initiative [23].

\subsection{Ten year mortality risk and absolute mortality}

For the secondary objective we estimated the mortality risk in patients with atherosclerotic CGI by estimating the ten-year risk of death due to CVD as well as calculating the all cause mortality as standardized mortality ratio [24]. We calculated the expected mortality during follow-up using age and sex specific mortality rates of the Dutch population. The ten-year risk of death due to CVD was determined for cases and controls by means of the SCORE risk chart [25]. For patients with a history of a cardiac event, in particular myocardial infarction or CABG, the PREDICT risk score [26] was used to estimate the ten-year risk of death due to CVD. This risk score system was originally developed for estimating the 6-year mortality risk after hospitalization for acute MI or unstable angina. The latter score system is based on factors such as age, ECG at the time of hospitalization, comorbidities and renal function. We estimated the ten-year mortality risk by deducing it from the 6 -year mortality. The PREDICT project showed that cancer mortality increased to $15 \%$ in
2-6 years. Subsequently, for estimating the ten-year mortality risk due to CVD alone, we corrected the calculated ten-year mortality risk for the $15 \%$ of estimated mortality risk due to cancer related mortality. Currently, there is no risk score for estimating the ten-year risk of mortality due to stroke. A recent cohort study reported the survival of stroke patients in the DUTCH transient ischemic attack Trial [22]. The cumulative risk of death at ten years was $47 \%$ for patients who presented with stroke at baseline and 34\% for those with TIA. We used these estimations for ten-year mortality in patients with a history of stroke or TIA. Thus, the median (IQR) estimated ten-year risk of death due to a cardiovascular event was assessed using the SCORE and PREDICT risk scores, while the mortality risk in patients with a history of a cerebrovascular event were based on data from the DUTCH transient ischemic attack trial [22].

\subsection{Statistical analyses}

Continuous data were described as mean (range), and median (interquartile range (IQR)) in case of skewed data, and percentages were given for categorical data. Mean values between patients with atherosclerotic CGI and controls were compared with student's 
T-test or Mann Whitney $U$-test and categorical data were compared with Chi-square test or Fisher's exact test. All cause mortality was calculated as standardized mortality ratio as described previously [24]. We calculated the 95 percent confidence interval of the standardized mortality ratio assuming a Poisson distribution of the observed number of deaths and using exact limits. A two-sided $p$ value below 0.05 was considered significant. Missing data of each variable were not included in the analysis for that specific variable. Crude and adjusted OR's and their 95\% confidence interval were estimated using logistic regression models.

\section{Results}

Between January 2006 and September 2009, a total of 195 patients were referred for evaluation of CGI; see Fig. 1. A consensus diagnosis of CGI was reached in 131 cases (67\% of all referred); 77 (59\%) had atherosclerotic CGI and were offered revascularization, After a median follow-up of 19 (interquartile range 9.50-27.50) months the definite diagnosis of atherosclerotic CGI was confirmed in 68 patients, i.e. these patients improved after treatment, and these were used as cases in this study; see Fig. 1. The remaining nine patients did not have symptom improvement after revascularization and therefore were not classified as having a definite diagnosis of atherosclerotic CGI. They were excluded from the analysis. From 68 patients who underwent revascularization: $59(87 \%)$ were treated with percutaneous revascularization, eight (12\%) had surgical revascularization, and one (1\%) patient died due to acute-on-chronic gastrointestinal ischemia before treatment. Autopsy results showed extensive gastrointestinal arterial atherosclerotic disease. There were no differences in age, gender, history of cardiovascular disease, and risk factors for atherosclerosis between patients who underwent percutaneous revascularization or surgery. Thirty-six (53\%) patients had single vessel stenosis, and $32(47 \%)$ patients had multi-vessel stenosis. The controls consisted of 132 subjects. Patients' and controls' characteristics are listed in Table 1 . Overall, $1.7 \%$ and $0.7 \%$ of required data concerning CGI cases and controls, respectively, were missing. This in particular pertained to systolic and diastolic blood pressure levels and glucose, total cholesterol, LDL-, HDL-cholesterol, and triglyceride levels.

\subsection{Univariate and multivariate analysis}

The healthy subjects of the DiaGene Study served as a control population in the current study. As mentioned in the Methods section, fasting blood samples were drawn from all controls after recruitment. Five percent of these controls were newly diagnosed with diabetes mellitus at inclusion. Diabetes, current smoking, history of CVD, hypercholesterolemia, family history of CVD, the use of cholesterol lowering agents, and anti-hypertensive agents were positively associated with atherosclerotic CGI. Females are more likely than males to have atherosclerotic CGI. The crude univariate analyses of the distribution of classical risk factors in patients with atherosclerotic CGI and controls are presented in Table 1 . The odds ratio's (OR) remained significant for female gender, diabetes and current smoking after further adjustment of the selected risk factors for gender, diabetes, hypercholesterolemia, current smoking, family history of CVD, and kidney function (see Table 2). The OR for CVD was attenuated after adjustment for gender (OR 21.61, 95\% confidence interval 8.40-55.55).

Atherosclerotic CGI cases had a median weight loss prior to referral of $9 \mathrm{~kg}$ (IQR 6-12 kg). The median period of weight loss was six (IQR 3.8-12.0) months. The BMI of patients with atherosclerotic CGI was median (IQR) $24.9(22.2-28.2)\left(\mathrm{kg} / \mathrm{m}^{2}\right)$ prior to the start of abdominal complaints, and it decreased to median $21.9(19.3-24.3)$ $\left(\mathrm{kg} / \mathrm{m}^{2}\right)$ at the time of the analysis. LDL-cholesterol levels were lower in patients with atherosclerotic CGI compared to controls (see Table 1). Similarly, patients with atherosclerotic CGI had lower systolic blood pressure levels than in the controls, although well within the normal range (data not shown). There was no difference in HDL-cholesterol, triglycerides and glucose between patients with atherosclerotic CGI and controls (data not shown).

There was no difference in the prevalence of risk factors for atherosclerosis between atherosclerotic CGI cases with singleversus multi-vessel stenosis (data not shown). The prevalence of risk factors between patients with atherosclerotic CGI and a history of CVD, and those without a history of CVD is presented in Table 3. Comparing the distribution of risk factors between male and female with atherosclerotic CGI, female had a significant lower BMI than male (median 21.1, IQR 17.6-23.3 vs. 23.5, IQR 21.1-27.2, $P<0.05$ ),

Table 1

Crude OR's in patients with atherosclerotic CGI and controls.

\begin{tabular}{|c|c|c|c|}
\hline Patient characteristics & CGI $n=68$ & Controls $n=132$ & Crude OR \\
\hline Age $^{a}$ & $67(57-75)$ & $66(63-72)$ & $0.98(0.94-1.00)$ \\
\hline Female gender & 47 (69\%) & $63(\%)$ & $2.45(1.32-4.55)^{* *}$ \\
\hline Diabetes & $14(21 \%)$ & $7(5 \%)$ & $4.63(1.77-12.11)^{* *}$ \\
\hline Hypercholesterolemia & $26(38 \%)$ & $32(24 \%)$ & $1.94(1.03-3.64)^{*}$ \\
\hline Hypertension & $46(68 \%)$ & $81(61 \%)$ & $1.32(0.71-2.44)$ \\
\hline \multicolumn{4}{|l|}{ Smoking } \\
\hline Never & $13(19 \%)$ & $52(39 \%)$ & 1.00 Reference \\
\hline Former & $25(37 \%)$ & $17(13 \%)$ & $1.91(0.90-4.02)$ \\
\hline Current & $30(44 \%)$ & $63(48 \%)$ & $5.88(2.48-14.00)^{* * *}$ \\
\hline CVD & $35(52 \%)$ & $7(5 \%)$ & $18.94(7.72-46.47)^{* * *}$ \\
\hline Family history of CVD & $35(52 \%)$ & $43(33 \%)$ & $2.20(1.21-4.00)^{* *}$ \\
\hline Cholesterol lowering agents & $32(47 \%)$ & $23(17 \%)$ & $4.21(2.19-8.11)^{* * *}$ \\
\hline Anti-hypertensive agents & $36(53 \%)$ & $39(30 \%)$ & $2.68(1.46-4.92)^{* * *}$ \\
\hline Current BMI $\left(\mathrm{kg} / \mathrm{m}^{2}\right)^{\mathrm{a}}$ & $21.9(19.3-24.3)$ & $25.8(24.1-28.1)$ & $0.76(0.69-0.84)^{* *}$ \\
\hline \multicolumn{4}{|l|}{ Kidney function ${ }^{\mathrm{b}}$} \\
\hline Normal or mildly & $53(78 \%)$ & $119(90 \%)$ & Reference \\
\hline decreased low & $15(22 \%)$ & $13(10 \%)$ & $2.59(1.15-5.83)^{*}$ \\
\hline Total cholesterol (mmol/l) & $4.3(3.6-5.0)$ & $5.2(4.7-5.9)$ & $0.43(0.31-0.61)^{* * *}$ \\
\hline LDL-cholesterol (mmol/l) & $2.2(1.8-2.8)$ & $3.5(3.0-4.0))$ & $0.27(0.17-0.42)^{* * *}$ \\
\hline
\end{tabular}

$\mathrm{CVD}=$ cardiovascular disease; BMI = body mass index; OR = odds ratio; OR is presented with 95\% CI Data are presented as mean (range), unless otherwise specified.

OR are reported with $95 \% \mathrm{CI}^{*}=P$-value $<0.05 ;^{* *}=P$-value $<0.01{ }^{* * *}=P$-value $<0.001$.

a Median (IQR 25-75).

b Normal or mildly decreased $=$ GFR $60 \geq 90 \mathrm{ml} / \mathrm{min} / 1.73 \mathrm{~m}^{2}$, Low $=$ GFR $<90 \mathrm{ml} / \mathrm{min} / 1.73 \mathrm{~m}^{2}$ Family history of premature cardiovascular disease is defined as cardiovascular disease in a first degree relative younger than 60 years. 
Table 2

Multivariate analysis of risk factors for atherosclerosis associated with atherosclerotic CGI

\begin{tabular}{lll}
\hline Patient characteristics & $\mathrm{OR}^{\mathrm{a}}$ & $95 \% \mathrm{CI}$ \\
\hline Female gender & 2.14 & $(1.05-4.36)^{*}$ \\
Diabetes & 5.59 & $(1.95-16.01)^{* *}$ \\
Hypercholesterolemia & 1.81 & $(0.88-3.74)$ \\
Hypertension & 1.20 & $(0.58-2.45)$ \\
Smoking & & \\
$\quad$ Never & 1.00 & Reference \\
Former & 1.71 & $(0.76-3.88)$ \\
$\quad$ Current & 5.78 & $(2.27-14.72)^{* *}$ \\
Family history of CVD & 1.94 & $(0.98-3.86)$ \\
Kidney function & & \\
$\quad$ Normal or mildly decreased & 1.00 & Reference \\
$\quad$ Low & 2.52 & $(0.98-6.49)$ \\
\hline
\end{tabular}

$\mathrm{CVD}=$ cardiovascular disease $; \mathrm{OR}=$ odds ratio $\mathrm{CI}=$ confidence interval.

a Adjusted for gender, diabetes, hypercholesterolemia, hypertension, current smoker, family history of CVD, and kidney function ${ }^{*}=P$-value $<0.05$; ${ }^{* *} P$-value $<0.001$; Family history of premature cardiovascular disease is defined as cardiovascular disease in a first degree relative younger than 60 years.

respectively. There were no other gender differences concerning the prevalence of risk factors.

\subsection{Mortality risk}

The data for estimating the death risk were available in 63 (93\%) out of 68 CGI cases, and 124 (94\%) out of 132 controls. The ten-year mortality risk due to cardiovascular disease was higher in CGI cases than controls, median (IQR) $8 \%(2-28)$ versus $5 \%(4-8), P=0.06$, respectively. The median (IQR) ten-year mortality risk in CGI cases $>55$ years was significantly higher than in controls, 15\% (5-37) versus $5 \%$ (4-8), respectively; $P=0.001$.

During a mean follow-up of 7.4 (range $0-31$ ) months, a total of 10 deaths were observed, this was equal to one death per 116 person-years in the CGI group. Three deaths took place within 3 months after the visit to our clinic as a result of acute gastrointestinal ischemia (percutaneous revascularization $=1$, surgery $=1$, and death before treatment $=1)$. The remaining seven patients (percutaneous revascularization $n=6$, surgery $n=1$ ) died due to cancer of respectively the sinus piriformis $(n=1)$, the small intestine $(n=1)$, and lung $(n=1)$, fatal myocardial infarction

Table 3

Patient characteristics and risk factors for atherosclerosis in atherosclerotic CGI patients with and without history of CVD.

\begin{tabular}{lcc}
\hline Patient characteristics & $\begin{array}{c}\text { With history of } \\
\text { CVD } n=35\end{array}$ & $\begin{array}{c}\text { Without history } \\
\text { of CVD } n=33\end{array}$ \\
\hline Age, years & $70(62-77)^{*}$ & $65(51-73)$ \\
Male gender & $14(40 \%)$ & $7(21 \%)$ \\
Diabetes & $12(34 \%)^{* *}$ & $2(6 \%)$ \\
Hypercholesterolemia & $18(51 \%)^{*}$ & $8(24 \%)$ \\
Hypertension & $27(77 \%)$ & $19(58 \%)$ \\
Smoking & & \\
$\quad$ Never & $5(14 \%)$ & $8(24 \%)$ \\
Former & $14(40 \%)$ & $11(33 \%)$ \\
$\quad$ Current & $16(46 \%)$ & $14(43 \%)$ \\
Family history of premature CVD & $20(57 \%)$ & $15(46 \%)$ \\
Cholesterol lowering agent & $23(66 \%)^{* *}$ & $9(27 \%)$ \\
Anti-hypertension agents & $24(69 \%)^{* *}$ & $12(36 \%)$ \\
Current BMI $\left(\mathrm{kg} / \mathrm{m}^{2}\right)$ & $22.6(20.3-267)$ & $21.3(18.0-23.3)$ \\
Total cholesterol (mmol/l) & $4.3(3.0-4.8)$ & $4.4(3.6-5.1)$ \\
LDL-cholesterol (mmol/l) & $2.1(1.7-2.8)$ & $2.4(1.9-3.9)$ \\
\hline
\end{tabular}

$\mathrm{CVD}=$ cardiovascular disease; BMI = body mass index; Data are presented as median (IQR 25-75), unless otherwise specified; $P$-values are for controls vs. atherosclerotic CGI; ${ }^{*} p<0.05,{ }^{* *} p<0.01$; Family history of premature cardiovascular disease is defined as cardiovascular disease in a first degree relative younger than 60 years. $(n=1)$, terminal heart failure $(n=2)$, and from an unknown cause $(n=1)$. The all cause mortality relative to the Dutch population standardized for age and calendar period was 3.55 (95 percent confidence interval, 1.70 to $6.52, P=0.001$ ). After excluding 3 deaths that took place within 3 months after the visit to our clinic as a result of acute gastrointestinal ischemia, we observed 7 deaths in 116 person-years: the SMR of these patients with atherosclerotic CGI was 2.48 (95 percent confidence interval, $1.01-5.11, P=0.025$ ). This severe excess mortality resulted of three patients dying from cancer (carcinoma of the sinus piriformis, adenocarcinoma of the small intestine, and lung cancer), one patient having a fatal MI, two having terminal heart failure, and one dying from an unknown cause.

\section{Discussion}

Our study firstly showed that atherosclerotic CGI is associated with classical risk factors for atherosclerosis. Current smoking, diabetes, hypercholesterolemia, history and family history of CVD, and use of cholesterol lowering agents and anti-hypertensive agents are more prevalent in patients with atherosclerotic CGI. After adjusting for multiple risk factors diabetes, current smoking, and previous CVD were significantly associated with atherosclerotic CGI. Female preponderance is confirmed and remains striking. Secondly, our study shows that patients over 55 years of age with atherosclerotic CGI have a high risk of ten-year cardiovascular mortality. This was confirmed during follow-up by severe excess mortality. Patients with atherosclerotic CGI are at risk for CVD, and this advocates the strong need to consider secondary preventive therapy in patients with atherosclerotic CGI

These results are even more striking considering the fact that most patients lost a substantial amount of weight before the baseline measurement. The measured levels of cholesterol, glucose and blood pressure in patients with atherosclerotic CGI were nearly normal. This difference can partly be explained by marked weight loss in most patients with CGI [1,27], as weight loss is the initial treatment option for hypercholesterolemia, hypertension and hyperglycaemia. Moreover, the use of cholesterol lowering agents and anti-hypertensive agents were significantly higher in patients with atherosclerotic CGI comparing to controls. Fifty-two percent of patients with atherosclerotic CGI had known CVD, and therefore they were already treated with cholesterol lowering agents and anti-hypertensive agents. Therefore, weight loss and higher use of statins in CGI patients, resulted in lower LDL-cholesterol levels (see Table 1). Similarly, the weight loss and higher use of anti-hypertensive drugs in CGI patients resulted in lower systolic blood pressure levels than in the controls, although well within the normal range (data not shown).

Previous case series comparing endovascular or surgical treatment for CGI, have reported a wide range of prevalences of risk factors for atherosclerosis, ranging from a 10-93\% prevalence of hypercholesterolemia, $64-92 \%$ of hypertension, and $36-72 \%$ of previous coronary artery disease [1,12-18]. This may have been due to several factors. Firstly, except for one study [16], patients with CACS were not excluded from the analysis of data in these study cohorts. Secondly, all studies so far were uncontrolled. We evaluated the risk factors for atherosclerosis systematically at the time of analysis in our study, including medical and family history, and laboratory evaluation. Moreover, we used an age-matched control group to compare the prevalence of risk factors for atherosclerosis.

The healthy control subjects of the DiaGene Study served as a control population in the current study. The controls were selected on absence of a known history of diabetes, yet $5 \%$ was newly diagnosed with diabetes at inclusion. This DiaGene cohort was comparable in age, BMI, and hypertension to two other population-based studies in the Netherlands: the ERGO study [28] 
which consists of residents of a suburb in Rotterdam, and The Hoorn Study [29] which is a separate cohort of older Dutch subjects. As such, the DiaGene cohort represents the age-matched general Dutch population.

A remarkable predominance of female gender in patients with atherosclerotic CGI was observed. This is in line with earlier reports of larger cohorts of CGI patients $[1,12,14,18]$. Earlier case series reveal high prevalence CACS in women [30-32], however we excluded patients with CACS in our study. The female preponderance with comparable CVD risk factors, suggests a female specific anatomical or other susceptibility for ischemia due to intestinal vascular narrowing. Possible explanations for the female preponderance in CGI patients could be that women with chronic abdominal pain are more often referred to medical specialists, or have less potential for collateral circulation and therefore are more prone to develop CGI.

Patients with atherosclerotic CGI had a marked excess mortality during follow-up. The severe excess mortality resulted from CVD and cancer, both related to risk factors for atherosclerosis such as smoking. This observed mortality was in line with the estimated risk scores. The ten-year risk of death in all patients was estimated by combining the SCORE, the PREDICT score, and the DUTCH transient ischemic Trial. For the PREDICT score, missing ECG data at the time of the primary CGI event can have resulted in an underestimation of 10 year mortality risk and thus have led to a risk underestimation in the cases. By combining the different risk scores, we have estimated that patients with atherosclerotic CGI are at higher risk of cardiovascular mortality than controls. Taken together, the risk scores seem to have produced valid high risk estimations, as illustrated by the observed excess mortality.

This study has several limitations. First, our study is a single center study. Second, patients evaluated for CGI usually represent a highly selected population. Likely, patients who had more severe atherosclerotic disease were more likely to be referred for evaluation. And most clinicians will only consider CGI after exclusion of a variety of other conditions. Our department has a different setting. During the past 6 years we have set up a referral program for patients suspected of CGI. The awareness among referring clinicians and the clinical expertise of our program lowers the threshold for referral and CGI evaluation, and thus reduces the selection process prior to referral. Third, it is a relatively small group for evaluating risk factors for atherosclerosis, the cases included 68 patients with atherosclerotic CGI. Larger studies are therefore needed to confirm these results. And fourth, the observational nature of the study is a limitation, but the incidence of CGI is so low that decades of patient inclusion would be required for a sufficiently powered randomized trial.

\section{Conclusions}

Patients with atherosclerotic CGI have a significant risk for cardiovascular mortality, resulting from more prevalent classical risk factors for atherosclerosis. Based on these results and previously reported cases series, we would advocate CVD preventive therapy consisting of personalized life-style optimalization, statins, antihypertensives on indication, and after successful revascularization and recovery of intestinal mucosal low dose aspirin should be considered. Future research, most likely observational, is needed to observe a positive effect of the secondary prevention therapy in these patients.

\section{Conflict of interest/funding}

None of the authors have a conflict of interest regarding this article. No funding was applied for this study.

\section{References}

[1] Mensink PB, van Petersen AS, Geelkerken RH, Otte JA, Huisman AB, Kolkman JJ. Clinical significance of splanchnic artery stenosis. Br J Surg 2006; 93:1377-82.

[2] Mensink PB, Moons LM, Kuipers EJ. Chronic gastrointestinal ischaemia: shifting paradigms. Gut 2011;60:722-37.

[3] Expert Panel on Detection EaToHBCiA. Executive summary of the third report of the National cholesterol Education program (NCEP) expert Panel on detection, evaluation, and treatment of high blood cholesterol in adults (adult treatment panel III). JAMA 2001;285:2486-97.

[4] Grundy SM, Brewer Jr HB, Cleeman JI, Smith Jr SC, Lenfant C. American heart A, National heart LaBI. Definition of metabolic syndrome: report of the National Heart, Lung, and Blood Institute/American Heart Association Conference on scientific issues related to definition. Circulation 2004;109:433-8.

[5] CAPRIE Steering Committee. A randomised, blinded, trial of clopidogrel versus aspirin in patients at risk of ischaemic events (CAPRIE). Lancet 1996;348: $1329-39$.

[6] Vliegenthart R, Hollander M, Breteler MM, et al. Stroke is associated with coronary calcification as detected by electron-beam CT: the Rotterdam coronary calcification study. Stroke 2002;33:462-5.

[7] Cotter G, Cannon $\mathrm{CP}$, McCabe $\mathrm{CH}$, et al. Prior peripheral arterial disease and cerebrovascular disease are independent predictors of adverse outcome in patients with acute coronary syndromes: are we doing enough? Results from the orbofiban in patients with unstable coronary syndromes-thrombolysis in myocardial infarction (OPUS-TIMI) 16 study. Am Heart J 2003;145:622-7.

[8] Bhatt DL, Peterson ED, Harrington RA, et al. Prior polyvascular disease: risk factor for adverse ischaemic outcomes in acute coronary syndromes. Eur Heart J 2009;30:1195-202.

[9] Criqui MH, Langer RD, Fronek A, et al. Mortality over a period of 10 years in patients with peripheral arterial disease. N Engl J Med 1992;326:381-6.

[10] Wijnhoud AD, Maasland L, Lingsma HF, Steyerberg EW, Koudstaal PJ Dippel DW. Prediction of major vascular events in patients with transient ischemic attack or ischemic stroke: a comparison of 7 models. Stroke 2010;41: 2178-85.

[11] Verouden NJ, Haeck JD, Kuijt WJ, et al. Early ST-segment recovery after primary percutaneous coronary intervention accurately predicts longterm prognosis after acute myocardial infarction. Am Heart J 2010;159: 1005-11.

[12] Kasirajan K, O’Hara PJ, Gray BH, et al. Chronic mesenteric ischemia: open surgery versus percutaneous angioplasty and stenting. J Vasc Surg 2001;33: 63-71.

[13] Silva JA, White CJ, Collins TJ, et al. Endovascular therapy for chronic mesenteric ischemia. J Am Coll Cardiol 2006;47:944-50.

[14] Mell MW, Acher CW, Hoch JR, Tefera G, Turnipseed WD. Outcomes after endarterectomy for chronic mesenteric ischemia. J Vasc Surg 2008;48: $1132-8$.

[15] Fioole B, van de Rest HJ, Meijer JR, et al. Percutaneous transluminal angioplasty and stenting as first-choice treatment in patients with chronic mesenteric ischemia. J Vasc Surg 2010;51:386-91.

[16] Kruger AJ, Walker PJ, Foster WJ, Jenkins JS, Boyne NS, Jenkins J. Open surgery for atherosclerotic chronic mesenteric ischemia. J Vasc Surg 2007; 46:941-5.

[17] Oderich GS, Bower TC, Sullivan TM, Bjarnason H, Cha S, Gloviczki P. Open versus endovascular revascularization for chronic mesenteric ischemia: riskstratified outcomes. J Vasc Surg 2009;49:1472-9. e3.

[18] Sarac TP, Altinel O, Kashyap V, et al. Endovascular treatment of stenotic and occluded visceral arteries for chronic mesenteric ischemia. J Vasc Surg 2008; 47:485-91.

[19] Voight BF, Scott LJ, Steinthorsdottir V, et al. Twelve type 2 diabetes susceptibility loci identified through large-scale association analysis. Nat Genet 2010;42:579-89.

[20] Mensink PB, Geelkerken RH, Huisman AB, Kuipers EJ, Kolkman JJ. Twenty-four hour tonometry in patients suspected of chronic gastrointestinal ischemia. Dig Dis Sci 2008;53:133-9.

[21] Sana A, Vergouwe Y, van Noord D, et al. Radiological imaging and gastrointestinal tonometry add value in diagnosis of chronic gastrointestinal ischemia. Clin Gastroenterol Hepatol 2011;9:234-41.

[22] van Wijk I, Kappelle LJ, van Gijn J, et al. Long-term survival and vascular event risk after transient ischaemic attack or minor ischaemic stroke: a cohort study. Lancet 2005;365:2098-104.

[23] Levey AS, Coresh J, Balk E, et al. National Kidney Foundation practice guidelines for chronic kidney disease: evaluation, classification, and stratification. Ann Intern Med 2003;139:137-47.

[24] Sijbrands EJ, Westendorp RG, Defesche JC, de Meier PH, Smelt AH, Kastelein JJ Mortality over two centuries in large pedigree with familial hypercholesterolaemia: family tree mortality study. BMJ 2001;322:1019-23.

[25] Conroy RM, Pyorala K, Fitzgerald AP, et al. Estimation of ten-year risk of fatal cardiovascular disease in Europe: the SCORE project. Eur Heart J 2003;24: 987-1003.

[26] Jacobs Jr DR, Kroenke C, Crow R, et al. PREDICT: a simple risk score for clinical severity and long-term prognosis after hospitalization for acute myocardial infarction or unstable angina: the Minnesota heart survey. Circulation 1999; 100:599-607. 
[27] Brandt LJ, Boley SJ. AGA technical review on intestinal ischemia. American Gastrointestinal Association. Gastroenterology 2000;118:954-68.

[28] Mennen LI, Witteman JC, Geleijnse JM, Stolk RP, Visser MC, Grobbee DE. Risk factors for cardiovascular diseases in the elderly; the ERGO study (Erasmus Rotterdam Health and the Elderly). Ned Tijdschr Geneeskd 1995;139:1983-8.

[29] de Vegt F, Dekker JM, Ruhe HG, et al. Hyperglycaemia is associated with allcause and cardiovascular mortality in the Hoorn population: the Hoorn Study. Diabetologia 1999;42:926-31.
[30] Gloviczki P, Duncan AA. Treatment of celiac artery compression syndrome: does it really exist? Perspect Vasc Surg Endovasc Ther 2007:19:259-63.

[31] Grotemeyer D, Duran M, Iskandar F, Blondin D, Nguyen K, Sandmann W. Median arcuate ligament syndrome: vascular surgical therapy and follow-up of 18 patients. Langenbecks Arch Surg 2009;394:1085-92.

[32] Mensink PB, van Petersen AS, Kolkman JJ, Otte JA, Huisman AB, Geelkerken RH. Gastric exercise tonometry: the key investigation in patients with suspected celiac artery compression syndrome. J Vasc Surg 2006;44:277-81. 